\title{
Testing Coefficients of Autoregressive Conditional Heteroskedasticity Models by Graphical Approach
}

\author{
Fengjing Cai ${ }^{\text {a, }}$, Yuan $\mathrm{Li}^{\text {b }}$ \\ ${ }^{a}$ School of Mathematics \& Information Science, Wenzhou University, Wenzhou, Zhejiang Province, China \\ ${ }^{b}$ School of Mathematics \& Information Science, Guangzhou University, Guangzhou, Guangdong Province, \\ China
}

\begin{abstract}
The graphical approach is applied to the autoregressive conditional heteroskedasticity time series models. After transformation, it is shown that the coefficients of GARCH model are the conditional correlation coefficients conditioned on the other components of the time series, then a new method is proposed to test the significance of the coefficients of GARCH model.
\end{abstract}

Index Terms: Time Series Chain Graph; ARCH; GARCH

(C) 2012 Published by MECS Publisher. Selection and/or peer review under responsibility of the Research Association of Modern Education and Computer Science.

\section{Introduction}

Financial economists are concerned with modelling dependence in asset returns. Observations in return series of financial assets observed at daily are uncorrelated or nearly uncorrelated, the series contain higherorder dependence. The models of autoregressive conditional heteroskedasticity (ARCH)[1] form the most popular way of parameterizing this dependence. Its generalization, the Generalized ARCH (GARCH) model[2] is a more parsimonious model of the conditional variance than a high-order ARCH model, most users prefer it to the simpler ARCH alternative.

Graphical models have become an important method for the analyzing multivariate data[3-6]. Recently they have been introduced to model dependence structures among multivariate time series[7-11]. Graphs to describe multivariate time series can be classified basically into the time series chain graph, partial correlation graph and Granger causality graph.

In this paper, we express the autoregressive conditional heteroskedasticity models as time chain graphical models. In time series chain models, each vertex in a graph represents a separate variable at fixed time, and an

\footnotetext{
* Corresponding author.

E-mail address: cyclie@163.com
} 
edge between two vertices denotes that the two variables have causal relations or dependence. The direction of an edge between two vertices is determined by both time and partial correlation. We show that the coefficient of GARCH model deformation is the partial correlation coefficient after removing the linear effects of the other components of the time series. A new procedure with graphical approach is proposed to test the coefficients of GARCH model.

\section{Time series chain graph and garch model}

We introduce a time series chain graph (TSC-graph) by firstly giving the definition of a graph over a finite and nonempty set $V$. Let $G=(V, E)$, elements in $V$ are called vertices, and $E$ is a set of directed or undirected edges denoted as $a \rightarrow b, a-b . a \rightarrow b$ is called directed edges, while $a-b$ is called the undirected edge.

Suppose that $X=\left(X_{1}(t), X_{2}(t), \cdots, X_{d}(t)\right)^{\prime}$ is a d-dimensional stationary time series. The TSC-graph of the stationary process $X$ is the graph $G_{T S}=\left(V_{T S}, E_{T S}\right)$ where $V_{T S}=V \times Z$ with $V=\{1,2, \cdots, d\}$ and $Z$ being an integer set, and the edge set $E_{T S}$ satisfies

1) $\quad(a, t-u) \rightarrow(b, t) \notin E_{T S} \Leftrightarrow u \leq 0$ or $X_{a}(t-u) \perp X_{b}(t) \mid \bar{X}(t) \backslash\left\{X_{a}(t-u)\right\}$

2) $\quad(a, t-u)-(b, t) \notin E_{T S} \Leftrightarrow u \neq 0$ or $X_{a}(t) \perp X_{b}(t) \mid \bar{X}_{V}(t) \cup\left\{X_{V \backslash\{a, b\}}(t)\right\}$

where $\bar{X}_{A}(t)=\left\{X_{A}(s), s<t\right\}$ denotes the past of the subprocess $X_{A}=\left\{X_{A}(t)\right\}$ at time $t$. Here it involves conditional orthogonality. For random vectors $X, Y$ and $Z$, we call $X$ and $Y$ to be conditionally orthogonal given $Z$, denoted by $X \perp Y \mid Z$, if $X$ and $Y$ are uncorrelated after the linear effects of $Z$ have been removed.

For stationary $\mathrm{ARCH}(\mathrm{q})$ model

$$
\left\{\begin{array}{l}
X_{t}=\sigma_{t} \varepsilon_{t} \\
\sigma_{t}^{2}=\alpha_{0}+\sum_{i=1}^{q} \alpha_{i} X_{t-i}^{2}
\end{array}\right.
$$

where $\left\{\varepsilon_{t}\right\}$ is i.i.d. Normal random with mean zero and variance 1 and $\alpha_{0}>0, \alpha_{i} \geq 0(i=1, \cdots, q)$.

Simple transformation is given as follows:

$$
X_{t}^{2}=\alpha_{0}+\sum_{i=1}^{q} \alpha_{i} X_{t-i}^{2}+\eta_{t}
$$

where $\eta_{t}=\sigma_{t}^{2}\left(\varepsilon_{t}^{2}-1\right)$ is uncorrelated time series with mean zero and variance $\lambda_{1}$.

Let $Y_{t}=X_{t}^{2}$, then

$$
Y_{t}=\alpha_{0}+\sum_{i=1}^{q} \alpha_{i} Y_{t-i}+\eta_{t}
$$

In order to verify the auto-regressive conditional heteroskedasticity, we need to test $H_{0}: \alpha_{i}=0(i=1, \cdots, q)$ via $H_{1}: \exists \alpha_{i} \neq 0$. So we test the significance of the coefficients for AR(q) model. 
Since we only deal with univariate time series, $V_{T S}$ in TSC-graph $G_{T S}=\left(V_{T S}, E_{T S}\right)$ is $Z=\{\cdots,-1,0,1, \cdots\}$, each vertex represents one component of $Y_{t}$ at some time. As an illustration, Fig. 1 presents a TSC graph $G_{T S}$ for $\operatorname{ARCH}(2)$ time series $Y_{t}$, where $t-2 \rightarrow t$ denotes that $Y_{t-2}$ is causal for $Y_{t}$ and an absent edge between $t-1$ and $t$ implies that there does not exist a causal relation between $Y_{t-1}$ and $Y_{t}$. The test of coefficients based on graphical approach for AR model has been discussed in detail[12].

$$
\begin{array}{ll}
t-2 \mathrm{O} \longrightarrow \mathrm{O} t \\
t-1 \quad 0
\end{array}
$$

Fig. 1 The graph for $\mathrm{ARCH}(2)$

For stationary $\operatorname{GARCH}(\mathrm{p}, \mathrm{q})$ model

$$
\left\{\begin{array}{l}
X_{t}=\sigma_{t} \varepsilon_{t} \\
\sigma_{t}^{2}=\alpha_{0}+\sum_{i=1}^{q} \alpha_{i} X_{t-i}^{2}+\sum_{i=1}^{p} \beta_{i} \sigma_{t-i}^{2}
\end{array}\right.
$$

where $\quad\left\{\varepsilon_{t}\right\}$ is i.i.d. Normal random with mean zero, variance 1 and $\alpha_{0}>0, \alpha_{i}, \beta_{j} \geq 0(i=1, \cdots, q, j=1, \cdots, p)$.

Let $Y_{t}=X_{t}^{2}, \varphi_{i}=\left\{\begin{array}{l}\alpha_{i}+\beta_{i}, 1 \leq i \leq \min (p, q) \\ \alpha_{i}, p<i \leq q \\ \beta_{i}, q<i \leq p\end{array}\right.$

Simple transformation is given as follows:

$$
Y_{t}=\alpha_{0}+\sum_{i=1}^{m} \varphi_{i} Y_{t-i}-\sum_{i=1}^{p} \beta_{i} \eta_{t-i}+\eta_{t}
$$

where $m=\max (p, q), \eta_{t}=\sigma_{t}^{2}\left(\varepsilon_{t}^{2}-1\right)$ is uncorrelated time series with mean zero and variance $\lambda_{2}$. The most popular GARCH model in applications has been the $\operatorname{GARCH}(1,1)$ model. For stationary $\operatorname{GARCH}(1,1)$

$$
\left\{\begin{array}{l}
X_{t}=\sigma_{t} \varepsilon_{t} \\
\sigma_{t}^{2}=\alpha_{0}+\alpha_{1} X_{t-1}^{2}+\beta_{1} \sigma_{t-1}^{2}
\end{array}\right.
$$

Simple transformation is given as follows:

$$
Y_{t}=\alpha_{0}+\varphi_{1} Y_{t-1}-\beta_{1} \eta_{t-1}+\eta_{t}
$$

where $\varphi_{1}=\alpha_{1}+\beta_{1}$.

Let $y_{1, t}=Y_{t}, y_{2, t}=\eta_{t}$,Fig. 2 presents a TSC graph $G_{T S}$ for model(2.7),Vertex $i$ in $V$ represents time series $y_{i, t}$, while vertex $(i, t-u)$ in TSC-graph $V_{T S}=V \times Z$ denotes the component $y_{i, t-u}$. We know from the definition of the TSC-graph that $(1, t-1) \rightarrow(1, t)$ denotes that $Y_{t-1}$ is causal for $Y_{t}$ and an absent edge between $(2, t-1)$ and $(1, t)$ implies that $\eta_{t-1}$ is noncausal for $Y_{t}$. 


$$
(1, t-1) \mathrm{O} \longrightarrow \mathrm{O}(1, t)
$$

$$
(2, t-1) \quad \circ
$$

Fig. 2 The graph for GARCH(1,1)

To verify the conditional heteroskedasticity, we need to test $H_{0}: \varphi_{i}=0$ for GARCH(p,q) model. So we need to test the significance of the coefficients for model (2.5).

\section{III. parametric tests by graphical models}

Let $\pi=\{\mu+\alpha U+\beta V \mid \mu, \alpha, \beta \in R\}$ be a linear subspace spanned by random variables $U$ and $V$. The best linear predictor, denoted by $\operatorname{Proj}_{\pi} X$, of $X$ on $U$ and $V$ is defined as

$E\left(X-\operatorname{Proj}_{\pi} X\right)^{2}=\min _{\mu, \alpha, \beta} E(X-\mu-\alpha U-\beta V)^{2}$

$\operatorname{Proj}_{\pi} X$ is called the linear projector of $X$ on $\pi$.The linear property holds for $\operatorname{Proj}_{\pi} X$.

\section{Proposition 3.1}

$\operatorname{Proj}_{\pi}(a X+b Y)=a \operatorname{Proj}_{\pi} X+b \operatorname{Proj}_{\pi} Y$

The definition of the linear projector $\operatorname{Proj}_{\pi} X$ can be generalized to a general linear subspace $\pi$ spanned by random variables $X_{1}, X_{2}, \cdots, X_{n}$. With $\operatorname{Proj}_{\pi} X$, we readily define the partial covariance as follows:

$\operatorname{Cov}_{L}(X, Y \mid \pi)=E\left(X-\operatorname{Proj}_{\pi} X\right)\left(Y-\operatorname{Proj}_{\pi} Y\right)$

Theorem 3.1 For model (2.5)

1) $(1, t-k) \rightarrow(1, t) \in E_{T S} \Leftrightarrow k \in\{1, \cdots, m\}$ and $\varphi_{k} \neq 0$.

2) $(2, t-k) \rightarrow(1, t) \in E_{T S} \Leftrightarrow k \in\{1, \cdots, p\}$ and $\beta_{k} \neq 0$.

1) Proof. We know from the definition of the TSC-graph that

$$
(1, t-k) \rightarrow(1, t) \notin E_{T S} \Leftrightarrow Y_{t-k} \perp Y_{t} \mid \pi^{(1,-k)}
$$

which is equivalent to $\operatorname{Cov}_{L}\left(Y_{t-k}, Y_{t} \mid \pi^{(1,-k)}\right)=0$, where $\pi^{(1,-k)}$ is a linear subspace spanned by $\left\{Z_{s}, s<t\right\}-\left\{Y_{t-k}\right\}$ with $Z_{t}=\left(Y_{t}, \eta_{t}\right)^{\prime}$. As $\operatorname{Proj}_{\pi^{(1,-k)}} \eta_{t}=0$, it follows from (3.2) and the linear property of $\operatorname{Proj}_{\pi} Y$ that $\operatorname{Proj}_{\pi^{(1,-k)}} Y_{t}=\sum_{\substack{j=1 \\ j \neq k}}^{m} \varphi_{j} Y_{t-j}-\sum_{j=1}^{p} \beta_{j} \eta_{t-j}+\varphi_{k} \cdot \operatorname{Proj}_{\pi^{(1,-k)}} Y_{t-k}$

Then we have

$$
\begin{aligned}
& \gamma_{k \mid \pi^{(1,-k)}}=\operatorname{Cov}_{L}\left(Y_{t-k}, Y_{t} \mid \pi^{(1,-k)}\right) \\
& =E\left(Y_{t}-\operatorname{Proj}_{\pi^{(1,-k)}} Y_{t}\right)\left(Y_{t-k}-\operatorname{Proj}_{\pi^{(1,-k)}} Y_{t-k}\right) \\
& =E\left\{\left(\varphi_{k}\left(Y_{t-k}-\operatorname{Proj}_{\pi^{(1,-k)}} Y_{t-k}\right)+\eta_{t}\right)\left(Y_{t-k}-\operatorname{Proj}_{\pi^{(1,-k)}} Y_{t-k}\right)\right\} \\
& =\varphi_{k} E\left\{\left(Y_{t-k}-\operatorname{Proj}_{\pi^{(1,-k)}} Y_{t-k}\right)^{2}\right\} \\
& =\varphi_{k} \operatorname{Var}\left(Y_{t-k} \mid \pi^{(1,-k)}\right)
\end{aligned}
$$


Then (3.3) follows from (3.5) directly.

2) Proof. We know from the definition of the TSC-graph that

$(2, t-k) \rightarrow(1, t) \notin E_{T S} \Leftrightarrow \eta_{t-k} \perp Y_{t} \mid \pi^{(2,-k)}$

which is equivalent to $\operatorname{Cov}_{L}\left(\eta_{t-k}, Y_{t} \mid \pi^{(2,-k)}\right)=0$, where $\pi^{(2,-k)}$ is a linear subspace spanned by $\left\{Z_{s}, s<t\right\}-\left\{\eta_{t-k}\right\}$.

Then we have

$$
\begin{aligned}
& \gamma_{k \mid \pi^{(2,-k)}}=\operatorname{Cov}_{L}\left(\eta_{t-k}, Y_{t} \mid \pi^{(2,-k)}\right) \\
& =E\left(Y_{t}-\operatorname{Proj}_{\pi^{(2,-k)}} Y_{t}\right)\left(\eta_{t-k}-\operatorname{Proj}_{\pi^{(2,-k)}} \eta_{t-k}\right) \\
& =E\left\{\left(\beta_{k}\left(\operatorname{Proj}_{\left.\pi^{(2,-k}\right)} \eta_{t-k}-\eta_{t-k}\right)+\eta_{t}\right)\left(Y_{t-k}-\operatorname{Proj}_{\pi^{(2,-k)}} Y_{t-k}\right)\right\} \\
& =-\beta_{k} E\left\{\left(\eta_{t-k}-\operatorname{Proj}_{\pi^{(2,-k)}} \eta_{t-k}\right)^{2}\right\} \\
& =-\beta_{k} \operatorname{Var}\left(\eta_{t-k} \mid \pi^{(2,-k)}\right)
\end{aligned}
$$

Then (3.4) follows from (3.6) directly.

Theorem 3.1 shows us that $Y_{t-l}$ is noncausal for $Y_{t}$, if and only if $\varphi_{l}=0$. Therefore if we want to test $\varphi_{l}=0$, we need only test if there exists a directed edge $(1, t-l) \rightarrow(1, t)$, based on which a parametric test is proposed. We know $\eta_{t-l}$ is causal for $Y_{t}$, if and only if $\beta_{l} \neq 0$. Therefore if we want to test $\beta_{l}=0$, we need only test if there exists a directed edge $(2, t-l) \rightarrow(1, t)$.

Based on sample $Y_{1}, Y_{2}, \cdots, Y_{n}$ from model (2.5), we only take into account the observed part of the past. Then the estimates of $\gamma_{k \mid \pi^{(1,-k)}}$ and $\gamma_{k \mid \pi^{(2,-k)}}$ is given as follows:

$$
\begin{aligned}
& \hat{\gamma}_{k \mid \pi^{(1,-k)}}=\operatorname{Cô} v_{L}\left(Y_{u-k}, Y_{u} \mid \pi_{u}{ }^{(1,-k)}\right) \\
& \hat{\gamma}_{k \mid \pi^{(2,-k)}}=\operatorname{Cô} v_{L}\left(\eta_{u-k}, Y_{u} \mid \pi_{u}^{(2,-k)}\right)
\end{aligned}
$$

where $\pi_{u}{ }^{(1,-k)}$ and $\pi_{u}{ }^{(2,-k)}$ is a subspace spanned respectively by $\{Z(m+1), \cdots, Z(u-1)\}-\left\{Y_{u-k}\right\}$ and $\{Z(m+1), \cdots, Z(u-1)\}-\left\{\eta_{u-k}\right\}$ for some fixed $u$.

Discussion of asymptotic properties and computation of $\gamma_{k \mid \pi^{(1,-k)}}$ and $\gamma_{k \mid \pi^{(2,-k)}}$ for a general GARCH(p,q) model are complicated. Next, as an illustration, we consider stationary $\operatorname{GARCH}(1,1)$ model. We need to test $H_{0}: \alpha_{1}+\beta_{1}=0$ via $H_{1}: \varphi_{1}>0$ and $H_{0}: \beta_{1}=0$ via $H_{1}: \beta_{1}>0$.

For some fixed $u>2$, we first compute

$$
\begin{aligned}
& \gamma_{1 \mid \pi^{(1,-1)}}=\operatorname{Cov}_{L}\left(Y_{u-1}, Y_{u} \mid \pi_{u}^{(1,-1)}\right) \\
& \gamma_{1 \mid \pi^{(2,-1)}}=\operatorname{Cov}_{L}\left(\eta_{u-1}, Y_{u} \mid \pi_{u}^{(2,-1)}\right)
\end{aligned}
$$

It is easily shown that

$$
\gamma_{1 \mid \pi^{(1,-1)}}=\gamma_{1}^{y}-\gamma_{1}^{y, \eta}
$$




$$
\gamma_{1 \mid \pi^{(2,-1)}}=\gamma_{1}^{y, \eta}-\frac{\gamma_{1}^{y} \cdot \gamma_{0}^{y, \eta}}{\gamma_{0}^{y}}
$$

where $\gamma_{k}^{y}=\operatorname{Cov}\left(Y_{t}, Y_{t-k}\right), \gamma_{k}^{y, \eta}=\operatorname{Cov}\left(Y_{t}, \eta_{t-k}\right), k=0,1$.

Then we know from (3.11) and (3.12) that $\gamma_{1 \mid \pi^{(1,-1)}}$ and $\gamma_{1 \mid \pi^{(2,-1)}}$ can be estimated by

$$
\begin{aligned}
& \hat{\gamma}_{1 \mid \pi^{(1,-1)}}=\hat{\gamma}_{1}^{y}-\hat{\gamma}_{1}^{y, \eta} \\
& \hat{\gamma}_{1 \mid \pi^{(2,-1)}}=\hat{\gamma}_{1}^{y, \eta}-\frac{\hat{\gamma}_{1}^{y} \cdot \hat{\gamma}_{0}^{y, \eta}}{\hat{\gamma}_{0}^{y}}
\end{aligned}
$$

where

$$
\hat{\gamma}_{k}^{y}=\frac{1}{n} \sum_{t=1}^{n-k}\left(Y_{t}-\bar{Y}\right)\left(Y_{t+k}-\bar{Y}\right), \hat{\gamma}_{k}^{y, \eta}=\frac{1}{n} \sum_{t=1}^{n-k}\left(Y_{t+k}-\bar{Y}\right)\left(\eta_{t}-\bar{\eta}\right) \text { with } \bar{Y}=\frac{1}{n} \sum_{t=1}^{n} Y_{t}, \bar{\eta}=\frac{1}{n} \sum_{t=1}^{n} \eta_{t} .
$$

Let

$$
A=\left(\begin{array}{lll}
\varphi_{1} & 0 & 0 \\
1 & 0 & 0 \\
0 & 1 & 0
\end{array}\right), B=\left(\begin{array}{rrr}
-\beta_{1} & 0 & 0 \\
0 & 0 & 0 \\
0 & 0 & 0
\end{array}\right), C=\left(\begin{array}{l}
1 \\
0 \\
0
\end{array}\right)
$$

and $\tilde{Y}_{t}=\left(Y_{t}, Y_{t-1}, Y_{t-2}\right)^{\prime}$.Then the state space representation of model (2.7) is as follows:

$$
\tilde{Y}_{t}=A \tilde{Y}_{t-1}+B \eta_{t-1}+C \eta_{t}
$$

Theorem 3.2 For model(2.7) with $Y_{t}=C^{\prime} \tilde{Y}_{t}$, suppose that $E \eta_{t}=0, E \eta_{t}^{3}=0, E \eta_{t}^{2}=\sigma^{2}<\infty$, $E \eta_{t}^{4}<\infty$ and $\rho\left(A \otimes A+\sigma^{2} B \otimes B\right)<1$. Then as $n \rightarrow \infty$, it holds that

1) $\hat{\gamma}_{k}^{y} \stackrel{p}{\longrightarrow} \gamma_{k}^{y}, n^{1 / 2}\left(\hat{\gamma}_{k}^{y}-\gamma_{k}^{y}\right) \stackrel{d}{\longrightarrow} N\left(0, \sigma_{k}^{2}\right)$.

where $\sigma_{k}^{2}=\lim _{n \rightarrow \infty} n E\left(\hat{\gamma}_{k}^{y}-\gamma_{k}^{y}\right)^{2}, k=0,1$.

2) $\hat{\gamma}_{k}^{y, \eta} \stackrel{p}{\longrightarrow} \gamma_{k}^{y, \eta}, n^{1 / 2}\left(\hat{\gamma}_{k}^{y, \eta}-\gamma_{k}^{y, \eta}\right) \stackrel{d}{\longrightarrow} N\left(0, \sigma_{k, y, \eta}^{2}\right)$

where $\sigma_{k, y, \eta}^{2}=\lim _{n \rightarrow \infty} n E\left(\hat{\gamma}_{k}^{y, \eta}-\gamma_{k}^{y, \eta}\right)^{2}, k=0,1$.

3) $n^{1 / 2}\left(\hat{\gamma}_{1 \mid \pi^{(1,-1)}}-\gamma_{1 \mid \tau^{(1,-1)}}\right) \stackrel{d}{\longrightarrow} N\left(0, \sigma_{2}^{2}\right)$

where $\sigma_{2}^{2}=\theta^{\prime} W \theta$ with $W=\lim _{n \rightarrow \infty} n E(\hat{\gamma}-\gamma)(\hat{\gamma}-\gamma)^{\prime}$,

where $\hat{\gamma}=\left(\hat{\gamma}_{0}^{y}, \hat{\gamma}_{1}^{y}, \hat{\gamma}_{0}^{y, \eta}, \hat{\gamma}_{1}^{y, \eta}\right), \gamma=\left(\gamma_{0}^{y}, \gamma_{1}^{y}, \gamma_{0}^{y, \eta}, \gamma_{1}^{y, \eta}\right)$.

and $\theta=(0,1,0,-1)^{\prime}$.

4) $n^{1 / 2}\left(\hat{\gamma}_{1 \mid \pi^{(2,-1)}}-\gamma_{1 \mid \pi^{(2,-1)}}\right) \stackrel{d}{\longrightarrow} N\left(0, \sigma_{3}^{2}\right)$

where $\sigma_{3}^{2}=\zeta^{\prime} W \zeta$, and $\zeta=\left(\zeta_{1}, \zeta_{2}, \zeta_{3}, \zeta_{4}\right)^{\prime}$ with 
$\zeta_{1}=-\frac{\hat{\gamma}_{1}^{y} \cdot \hat{\gamma}_{0}^{y, \eta}}{\left(\hat{\gamma}_{0}^{y}\right)^{2}}, \quad \zeta_{2}=-\frac{\hat{\gamma}_{1}^{y, \eta}}{\hat{\gamma}_{0}^{y}}, \quad \zeta_{3}=-\frac{\hat{\gamma}_{1}^{y}}{\hat{\gamma}_{0}^{y}}, \quad \zeta_{4}=1$.

Theorem 3.2 can be directly proved by Shao[13] and Liu[14]. So we omit it here. Denote

$T_{1, n}=\frac{n^{1 / 2} \hat{\gamma}_{1 \mid \pi^{(1,-1)}}}{\sigma_{2}}$.

We know that under $H_{0}: \alpha_{1}=\beta_{1}=0, \gamma_{1 \mid \pi^{(1,-1)}}=0$.Hence it follows from Theorem 3.2 that $T_{1, n} \stackrel{d}{\longrightarrow} N(0,1)$, as $n \rightarrow \infty$. Therefore we reject $H_{0}: \alpha_{1}=\beta_{1}=0$ when $T_{1, n}>\Phi^{-1}(1-\alpha)$. Denote $T_{2, n}=\frac{n^{1 / 2} \hat{\gamma}_{1 \mid \pi^{(2,-1)}}}{\sigma_{3}}$

We know that under $H_{0}: \beta_{1}=0, \gamma_{1 \mid \pi^{(2,-1)}}=0$.Hence it follows from Theorem 3.2 that $T_{2, n} \stackrel{d}{\longrightarrow} N(0,1)$, as $n \rightarrow \infty$. Therefore we reject $H_{0}: \beta_{1}=0$ when $T_{2, n}<-\Phi^{-1}(1-\alpha)$.

We have to calculate $\gamma_{1 \mid \pi^{(1,-1)}}$ and $\gamma_{1 \mid \pi^{(2,-1)}}$ which involves calculating $\eta_{t}, \eta_{t}$ can be estimated by firstly estimating parameters $\alpha_{1}$ and $\beta_{1}$, then recursively by

Step 1. Regress $Y_{t}$ on $Y_{t-1}, t=2,3, \cdots, n$ and obtain $\hat{a}_{0}, \hat{a}_{1}$, and residuals

$e_{t}=Y_{t}-\hat{a}_{0}-\hat{a}_{1} Y_{t-1}, t=2,3, \cdots, n$.

Step 2. Regress $e_{t-1}$ on $Y_{t-1}, t=2,3, \cdots, n$ and obtain $\hat{b}_{0}, \hat{b}_{1}$ and residuals

$v_{t}=e_{t-1}-\hat{b}_{0}-\hat{b}_{1} Y_{t-1}, t=3,4, \cdots, n$.

Step 3. $\hat{\gamma}_{1 \mid \pi^{(2,-1)}}$ is given by

$\hat{\gamma}_{1 \mid \pi^{(2,-1)}}=\frac{1}{n-3} \sum_{t=4}^{n} e_{t} v_{t}$

\section{Conclusion}

The new test method based on the graphical approach for the conditional heteroskedasticity model is proposed in the paper. For an illustration, $\operatorname{GARCH}(1,1)$ is expressed the time series chain graphs and the test of the significance of the coefficients is given. Compared to the traditional test method, our methods are intuitive and very simple in computations. The new method will have important implication in the financial market.

\section{References}

[1] Engle R. F. "Autoregressive conditional heteroskedasticity with estimates of the variance of United Kingdom inflation”, Econometrica, Vol 50,No. 4, pp. 987-1007, 1982.

[2] Bollerslev T. "Generalized autoregressive conditional heteroskedasticity” Journal of Econometrics, Vol. 
31, No. 3, pp. 307-327,1986.

[3] Whittaker J. "Graphical models in applied multivariate statistics”, New York:Wiley, pp.1-120,1990.

[4] Cox D.R., Wermuth N. "Linear dependencies represented by chain graphs", Statistical Science, Vol. 8,No. 3, pp.204-218,1993.

[5] Edwards D. "Introduction to graphical modelling", New York: Springer, pp.1-20,2001.

[6] Lauritzen S.L. "Graphical models", Oxford: Oxford University Press, pp.1-33,1996.

[7] Reale M. “A graphical modelling approach to time series”, Lancaster University, pp.1-143,1998.

[8] Eichler M. “Graphical models in time series analysis”, University Heidelberg, pp.1-100, 1999.

[9] Dahlhaus R. "Graphical interaction models for multivariate time series", Metrika, Vol. 51, No. 2, pp. 157 172, 2000.

[10] Gather U., Imhoff M., Fried R. "Graphical models for multivariate time series from intensive care monitoring”, Statistics in Medicine, Vol. 21,No. 18, pp. 2685-2701,2002.

[11] Eichler M. "Granger causality and path diagrams for multivariate time series”,Journal of Econometrics, Vol. 137, No. 2, pp.334-353, 2007.

[12] IP W.C., Wong H., Li Y., Luo X.H. "Testing coefficients of AR and bilinear time series models by a graphical approach", Science in China Series A: Mathematics, Vol. 51, No. 12,pp. 2304-2314, 2008.

[13] Shao J. "Mathematical statistics”,New York: Springer-Verlag, pp.1-530,1999.

[14] Liu J. "On stationarity and asymptotic inference of bilinear time series models", Statist Sin,Vol. 2, pp.479-494,1992 